\title{
ATP-dependent chromatosome remodeling
}

\author{
Verena K. Maier, Mariacristina Chioda and \\ Peter B. Becker*
}

Adolf-Butenandt Institut, Abt. Molekularbiologie, and Münchner Zentrum für Integrierte Proteinforschung, Ludwig-Maximilian-Universität München, Schillerstrasse 44, D-80336 München, Germany

${ }^{*}$ Corresponding author

e-mail: pbecker@med.uni-muenchen.de

\begin{abstract}
Chromatin serves to package, protect and organize the complex eukaryotic genomes to assure their stable inheritance over many cell generations. At the same time, chromatin must be dynamic to allow continued use of DNA during a cell's lifetime. One important principle that endows chromatin with flexibility involves ATPdependent 'remodeling' factors, which alter DNA-histone interactions to form, disrupt or move nucleosomes. Remodeling is well documented at the nucleosomal level, but little is known about the action of remodeling factors in a more physiological chromatin environment. Recent findings suggest that some remodeling machines can reorganize even folded chromatin fibers containing the linker histone $\mathrm{H} 1$, extending the potential scope of remodeling reactions to the bulk of euchromatin.
\end{abstract}

Keywords: ATPase; chromatin; linker histone; nucleosome sliding.

\section{Introduction}

The former view of chromatin as a static entity has gradually been replaced by the concept of a highly dynamic structure, where components are continuously exchanged and which can be condensed and decondensed to adapt to the varying needs of the cell. One class of enzymes largely contributing to the dynamic nature of chromatin is represented by ATP-dependent nucleosome remodeling factors. Members of this enzyme family couple the hydrolysis of ATP to disruption of DNAhistone contacts. All remodeling factors contain an ATPase belonging to the SWI/SNF-family, which in most cases is associated with additional subunits to form large protein complexes (Flaus and Owen-Hughes, 2004). The outcome of the remodeling reaction depends on the specific features of the remodeling ATPase and the nature of the regulatory or targeting subunits it associates with. It ranges from complete disassembly of nucleosomes (their eviction) to the partial disassembly of histone octamers that provides an opportunity for the incorporation of histone variants and the repositioning of nucleosomes along
DNA, also referred to as 'nucleosome sliding' (Becker and Horz, 2002; Saha et al., 2006). Nucleosome remodeling factors may be targeted to specific loci by interaction with DNA-bound regulators, such as transcription factors, to disassemble or move just a single or very few nucleosomes at strategic sites (Mellor, 2005). Their frequent function as transcription co-factors explains their important contribution to defining gene expression programs and hence all aspects of cell fate. Consequently, impaired nucleosome remodeling may lead to deregulation and disease (de la Serna et al., 2006).

Conceivably, certain remodeling factors may also perform untargeted remodeling reactions throughout large chromosomal domains, effectively creating a dynamic 'ground state' of chromatin 'fluidity' that underlies all chromatin structure transitions. A global action of this kind is difficult to document and is largely hypothetical to date. Whether or not remodeling factors are likely to perform global functions in the nucleus depends very much on the extent to which they are able to act on their nucleosome substrate if buried in the complex structure that characterizes chromatin in vivo. To a first approximation, chromatin of interphase chromosomes consists of folded nucleosomal arrays containing the linker histone $\mathrm{H} 1$. The precise structure of the first level of folding of the nucleosomal array, the $30 \mathrm{~nm}$ fiber, is still somewhat controversial (Robinson and Rhodes, 2006). It is difficult to see how nucleosome remodeling may work in the presence of linker histones and in a folded nucleosome array. However, recent results from a variety of unrelated experimental approaches, which we will summarize in what follows, suggest that the theme of ATPdependent nucleosome remodeling may be extended to also include the reorganization of linker histone-DNA interactions and to folded chromatin.

\section{Linker histones and chromatin rigidity}

Folding of the nucleosomal array into fibers of approximately $30 \mathrm{~nm}$ in diameter is promoted by linker histones (Robinson and Rhodes, 2006). These histones are much less evolutionary conserved than the core histones and in many organisms exist as different variants (Izzo et al., 2008), which we refer to collectively as ' $\mathrm{H} 1$ ' unless indicated otherwise. A typical $\mathrm{H} 1$ consists of a structured globular domain and extended $\mathrm{N}$ - and C-terminal 'tail' domains. A linker histone may interact asymmetrically with a nucleosome to form a so-called 'chromatosome'. The globular domain binds nucleosomal DNA at the nucleosome dyad and asymmetrically at one site of entry into the nucleosome, whereas the highly positively charged C-terminus presumably extends to contact linker DNA (Brown et al., 2006). This combined interaction 
seals the DNA that wraps around the histone octamer, prevents its transient dissociation and thus 'locks-in' the nucleosome (Bednar et al., 1998). At the same time, H1 interaction leads to stabilization of the $30 \mathrm{~nm}$ fiber. The two current models for the organization of the $30 \mathrm{~nm}$ fiber place the linker DNA and the linker histone in the center of the fiber (Robinson and Rhodes, 2006).

To fulfill its known role in chromatin organization, $\mathrm{H} 1$ does not have to associate stably with chromatin. In fact, fluorescence recovery after photobleaching experiments revealed that, in vivo, $\mathrm{H} 1$ is surprisingly dynamic. The average residence time of $\mathrm{H} 1$ on one binding site was estimated to be approximately $3 \mathrm{~min}$. Although this means that $\mathrm{H} 1$ is less mobile than most chromatin-associated proteins, it exchanges considerably faster than the core histones, which on average stay bound to one site for several hours (Catez et al., 2006). An obvious question that arises from these observations is whether the dynamic exchange of $\mathrm{H} 1$ interactions is intrinsic or aided by other factors. The observation of a highly dynamic population of linker histones in embryonic stem cells that is absent in differentiated mouse erythroleukemia (MEL) cells (Meshorer et al., 2006; Yellajoshyula and Brown, 2006) maintains that linker histone dynamics is regulated by extrinsic factors. Histone chaperones may contribute to this process (see below). Could ATP-dependent remodeling, which is a major determinant of core histone dynamics, also be involved?

Up until very recently, the available evidence argued against a role for nucleosome remodeling in $\mathrm{H} 1$ dynamics. For several reasons, $\mathrm{H} 1$ was mainly considered an obstacle for chromatin remodeling factors. First, interaction of $\mathrm{H} 1$ with nucleosomes stabilizes the structure, restricts the flexibility of the linker DNA and hinders loosening of histone DNA contacts, a step required for all remodeling events (Saha et al., 2006). Chromatosomes show a much-reduced thermal mobility compared to nucleosomes (Pennings et al., 1994). Second, it limits the access of remodeling factors towards linker DNA by two means: by promoting folding of chromatin into the $30 \mathrm{~nm}$ fiber, where linker DNA is placed inside the fiber (Robinson and Rhodes, 2006), and by competing for a segment of linker DNA that is a strategic contact for nucleosome remodeling enzymes. Nuclease protection and crosslinking experiments revealed similar sites of interaction for $\mathrm{H} 1$ and the remodeling ATPase ISWI (Robinson and Rhodes, 2006; Saha et al., 2006). Considering that the bulk of $\mathrm{H} 1$ is associated with chromatin most of the time, one would therefore expect it to counteract ATP-dependent remodeling.

\section{ATP-dependent remodeling of H1-containing chromatin}

In agreement with the concerns raised above, the experimental evidence so far has highlighted the repressive nature of linker histones towards ATP-dependent remodeling. Remodeling mononucleosomes by the yeast SWI/ SNF complex, a large complex involved in regulating transcription, and dinucleosome remodeling by ACF were partially inhibited if $\mathrm{H} 1$ was bound to the substrate
(Hill and Imbalzano, 2000; Saeki et al., 2005). ACF is a dimeric remodeling machinery, in which the ATPase ISWI is associated with a large subunit, ACF1, which may serve regulatory or targeting functions (Ito et al., 1999). The specialized avian linker histone variant H5 furthermore inhibited remodeling of short nucleosome arrays by various remodeling factors (Horn et al., 2002).

However, there are also reports hinting that nucleosomes can be rendered mobile, even in the presence of H1. Ramachandran et al. concluded from experiments with single nucleosome and chromatosome particles that SWI/SNF-dependent repositioning of histone octamers occurred in the presence of $\mathrm{H} 1$ (Ramachandran et al., 2003). Furthermore, early experiments by Varga-Weisz et al. (1995) showed that nucleosomes could be moved by an ATP-dependent process, even within chromatin arrays fully loaded with $\mathrm{H} 1$. These arrays represent a much better approximation of physiological chromatin than single nucleosomes. However, because these experiments had been performed with crude Drosophila embryonic extracts, no conclusions about the factor requirements were possible.

We recently reinvestigated the effect of linker histones on nucleosome sliding by ACF in a well-defined system consisting of short chromatin fibers reconstituted from purified or recombinant components. We found that ACF or its ATPase ISWI alone were able to significantly increase the access to DNA packaged in these arrays, even if they were saturated with linker histones $\mathrm{H} 1$ or $\mathrm{H} 5$ (Maier et al., 2007). Moreover, ACF catalyzed the repositioning of entire chromatosomes within these arrays (Figure 1d,g).

The potential of ATP-dependent remodeling to generate nucleosome-free regions in chromatin has received much attention due to its obvious impact on gene activation. However, it is only recently appreciated that remodeling reactions are, in principle, reversible. Nucleosome sliding may be used to generate a nucleosomefree region or conversely, to close such a gap effectively restoring the integrity of the chromatin fiber (Figure 1b,e). Because the integrity of the nucleosomal array is prerequisite to fiber folding (Figure 1b-d), this type of remodeling leads to enhanced packaging of DNA and reduced accessibility. The ability to catalyze nucleosome sliding to distribute nucleosomes evenly on DNA, the 'nucleosome spacing activity', is a feature of some ISWI-containing nucleosome remodeling factors, such as CHRAC and ACF (Langst and Becker, 2001). Nucleosome spacing converts irregular successions of nucleosomes into fibers with regular nucleosome distances during in vitro chromatin assembly reactions (Becker, 2002) (see Figure $1 e, b)$. In the context of a chromatin assembly reaction, CHRAC and ACF can thus be considered reconstitution factors. Interestingly, there is mounting evidence that also local nucleosome eviction and its opposite, nucleosome deposition, may be catalyzed by the same ATPdependent remodelers in vivo (Mellor, 2005). In vitro, the nucleosome assembly reaction can be tuned to include proper deposition of the linker histone $\mathrm{H} 1$. Lusser et al. showed that different remodeling factors are able to deal with the challenge posed by the linker histone to different extents (Lusser et al., 2005). They found that in the pres- 


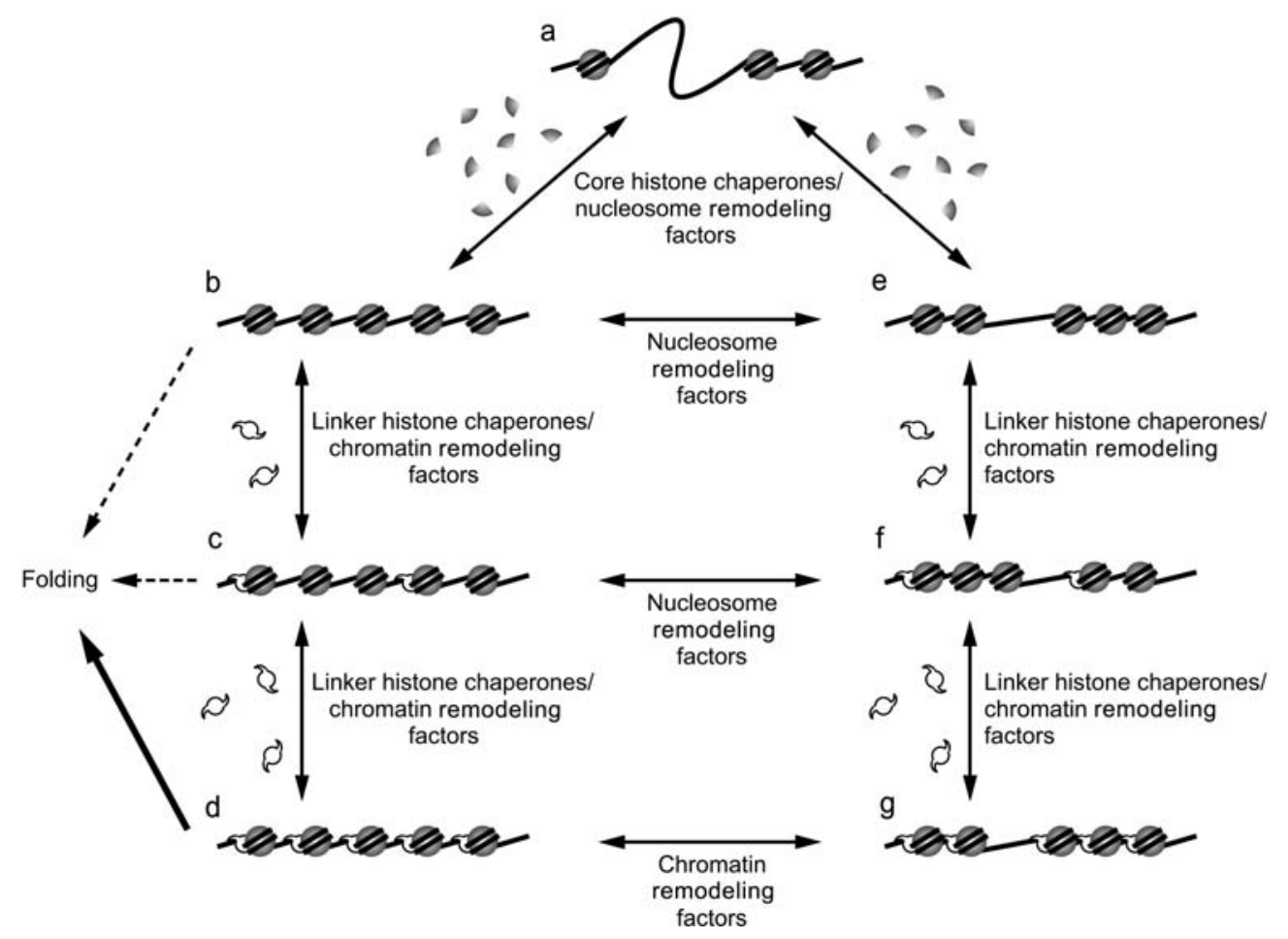

Figure 1 Interconversion of chromatin states through the interplay of core and linker histone chaperones and ATP-dependent remodeling factors.

The scenarios describe states of chromatin that differ in the regularity of the chromatin fiber and in core and linker histone stoichiometry. Spheres depict histone octamers around which the DNA (black line) is wound to form nucleosomes. Linker histones are represented in white. (a) Nucleosome fiber containing a nucleosome-free gap; (b) regularly spaced nucleosome array; (c) regularly spaced nucleosome array containing substoichiometric levels of $\mathrm{H} 1$; (d) regularly spaced chromatosome array with saturating linker histone levels; (e) irregular succession of nucleosomes that may arise after nucleosome assembly or if a nucleosome-free region is generated by nucleosome sliding; (f) nucleosome array containing substoichiometric levels of $\mathrm{H} 1$, where the nucleosomes devoid of $\mathrm{H} 1$ have been selectively moved; (g) irregularly spaced chromatosome arrays that may arise when chromatosomes are moved on DNA. Histone chaperones and ATP-dependent remodeling factors can act in concert to assemble core and linker histones onto DNA $(a-e)$. Binding linker histones allows remodeling by chromatin remodeling factors, but not nucleosome remodeling factors $(b, d, e, g)$. If present in substoichiometric amounts, linker histones may determine which nucleosomes are repositioned preferentially. Sliding may also be directional in the presence of $\mathrm{H} 1(\mathrm{c}, \mathrm{f})$. For further explanations, see text.

ence of the generic histone chaperone NAP-1 (see below) ACF was able to promote the formation of $\mathrm{H} 1$ containing nucleosome arrays, whereas the remodeling ATPase CHD1 was unable to incorporate $\mathrm{H} 1$, although it was perfectly able to help assemble nucleosome arrays.

We have now compared the effect of linker histones on the ability of ACF and CHD1 to render DNA in chromatin accessible. In our assay, linker histones almost entirely inhibited the remodeling activity of CHD1, whereas ACF remained surprisingly active (Maier et al., 2007). Because the presence of $\mathrm{H} 1$ affects both chromatin assembly and remodeling activities of these enzymes in similar ways, the two processes might be mechanistically linked. Apparently, nucleosome-remodeling factors differ in their abilities to cope with linker histone-containing chromatin and it remains to be investigated how $\mathrm{H} 1$ affects other remodeling factors. We suggest to distinguish between nucleosome remodelers, which only remodel $\mathrm{H} 1$-free nucleosomes (Figure $1 \mathrm{~b}, \mathrm{e}$ and a,b) and those enzymes that also work on $\mathrm{H} 1$-containing chromatin (Figure 1d,g and g,f), which we would like to call 'chromatin remodeling factors' to indicate the difference. While these concepts are being derived mainly from in vitro studies, Corona and colleagues recently found a connection between the activity of an ISWI-containing remodeling complex and $\mathrm{H} 1$ homeostasis in chromosomes (Corona et al., 2007) (see below).

\section{Effectors of $\mathrm{H} 1$ dynamics}

These very recent observations suggest that ATPdependent remodeling may contribute to the dynamic interaction of $\mathrm{H} 1$ with chromatin. However, nothing is known about the circumstances that govern chromatin remodeling. Nucleosome remodeling, by contrast, is intrinsic to the nature of the histone-DNA interaction and known to be modulated by the presence of histone chaperones that synergize with remodeling machines, by post-translational modifications of the histones, as well as by the targeting of dedicated remodeling machines to defined loci. Reviewing the parameters that govern linker histone dynamics may thus reveal functional interaction of ATP-dependent remodeling with other principles.

Due to the extensive wrapping of DNA around histone octamers, nucleosomes are very stable structures. However, fast and reversible detachments of large segments of DNA from the histone octamer surface driven by thermal energy can be readily observed (Anderson et al., 2002). The fact that histone-DNA interactions are intrin- 
sically dynamic may be prerequisite for the mechanism of active remodeling. Likewise, the extent to which ATPdependent remodeling contributes to rendering $\mathrm{H} 1$-chromatin interactions dynamic is presumably influenced by the intrinsic affinity of linker histones. This affinity may be tuned by incorporation of specialized linker histone variants. Different subtypes of $\mathrm{H} 1$ are found in many organisms. For example, mammals express at least six somatic variants $\left(\mathrm{H} 1 \mathrm{a}-\mathrm{e}\right.$ and $\left.\mathrm{H} 1^{\circ}\right)$, the oocyte-specific $\mathrm{H} 100$ and the testis-specific H1t. Genetic experiments in mice reveal a high degree of functional redundancy of these variants (Fan et al., 2005); however, their affinities for chromatin differ considerably. The strongest chromatin-binder among the somatic variants, $\mathrm{H} 1 \mathrm{~d}$, exhibits a 19-fold higher affinity than the weakest one, $\mathrm{H} 1 \mathrm{a}$ (Orrego et al., 2007). These differences in affinities may account for the observed distinct effects of these variants on gene expression and chromatin structure (see review by Izzo et al., 2008). An extreme example for how a linker histone variant controls chromatin condensation is provided by $\mathrm{H} 5$. $\mathrm{H} 5$ is found in avian erythrocytes, where it contributes to the transcriptionally inactive state characteristic of these cells. Because $\mathrm{H} 5$ binds to chromatin with a higher affinity than somatic $\mathrm{H} 1$, it has been the preferred variant for structural studies involving linker histones. Modeling the structure of the globular domain of $\mathrm{H} 5$ onto the nucleosome has revealed that unlike $\mathrm{H} 1, \mathrm{H} 5$ has the propensity to form dimers when bound to chromatin, which might contribute to the observed enhanced condensation (Fan and Roberts, 2006). In vitro, H5 inhibits chromatin remodeling by ACF to a larger degree than $\mathrm{H1}$, but not entirely (Maier et al., 2007).

While nucleosome sliding is achieved by nucleosome remodeling factors alone, nucleosome eviction and assembly usually requires the cooperation of dedicated histone chaperones serving as histone donors or acceptors (Figure 1a,b,e) (Tyler, 2002; Loyola and Almouzni, 2004; Workman, 2006). Prominent examples include the functional cooperation of the yeast-remodeling factor RSC and the well-known chaperone NAP-1 in nucleosome disassembly in vitro (Lorch et al., 2006). The histone chaperone nucleoplasmin, on the other hand, can facilitate nucleosome mobilization by SWI/SNF and ACF (Figure 1a,b,e) (Angelov et al., 2006).

In analogy, also linker histone chaperones may contribute to $\mathrm{H} 1$ dynamics. So far, the only report of synergism between a histone chaperone and a remodeler in modulating $\mathrm{H} 1$-chromatin interactions is the case of the assembly of $\mathrm{H} 1$-containing nucleosomal arrays (Figure $1 \mathrm{a}-\mathrm{d}$ ) by ACF and NAP-1, mentioned above (Lusser et al., 2005). NAP-1 is a versatile histone chaperone (Loyola and Almouzni, 2004) that can be used to deposit $\mathrm{H} 1$ onto nucleosomes, as well as to remove $\mathrm{H} 1$ from chromatin fibers, and thereby promote their decondensation (Mazurkiewicz et al., 2006). A handful of other $\mathrm{H} 1$ interactors are candidates for $\mathrm{H} 1$ chaperones. Nucleoplasmin, an abundant nuclear protein in Xenopus oocytes, removes somatic linker histone variants from chromatin and thereby facilitates transcription (Dimitrov and Wolffe, 1996). $\mathrm{H} 1$ assembly, on the other hand, might be fulfilled by the nuclear autoantigenic sperm protein, which is essential for cell proliferation and development and known to transport $\mathrm{H} 1$ into the nucleus (Richardson et al., 2006). A presumably more subtle role in controlling interaction of linker histones with chromatin is played by prothymosin $\alpha$, which modulates the association of $\mathrm{H} 1$ with chromatin (Karetsou et al., 1998). The functional interactions of these chaperones with ATP-dependent remodelers are not known.

Another principle known to influence nucleosome dynamics is the modification of histones at their $\mathrm{N}$ terminal domains. This may affect recognition by the remodeling machinery directly or indirectly through alteration of interactions that determine the folding of the nucleosomal array. For example, acetylation of lysine 16 at histone $\mathrm{H} 4$ (H4K16ac) inhibits fiber folding, as well as the ATPase activity of ISWI (Clapier et al., 2002; ShogrenKnaak and Peterson, 2006). Linker histones have been found to be phosphorylated, methylated, acetylated, ubiquitinated and formylated (Wisniewski et al., 2007); the best studied modification being their C-terminal phosphorylation. Phosphorylated linker histones are associated to mitotic chromosomes and concomitantly, overall phosphorylation increases during mitosis (Zlatanova et al., 2000). In addition, phosphorylation of linker histone regulates transcription and reduces their affinity to chromatin (Catez et al., 2006). This is also reflected by the fact that phosphorylation releases the inhibitory effect of H5 on chromatin remodeling (Horn et al., 2002). Expression of $\mathrm{H} 1$ mutants mimicking a constitutively phosphorylated state counteracts differentiation of MEL cells, even though they accumulated to only $20 \%$ of total $\mathrm{H} 1$ amount. Moreover, in these cells, not only the mutant $\mathrm{H} 1$ but also the endogenous $\mathrm{H} 1$ exhibited an increased mobility. This means that a mutation that prevents the interaction of the $\mathrm{H} 1 \mathrm{C}$-terminus with linker DNA, but still allows binding via the globular domain, may disrupt chromatin folding globally and hence affect the interactions of wild type linker histones as well (Yellajoshyula and Brown, 2006). The observation that $\mathrm{H} 1$ variants exhibit differences in their modification pattern adds another level of complexity for regulating their binding to chromatin (Wisniewski et al., 2007). An example for the effect of site-specific $\mathrm{H} 1$ methylation on chromatin interactions is given by the interaction of heterochromatin protein 1 with K26 methylated H1 (Daujat et al., 2005). This interaction may incorporate $\mathrm{H} 1$ into the structural network that characterizes heterochromatin and hence affect its mobility indirectly.

The local or global interactions of $\mathrm{H} 1$ with chromatin are not only modulated by the intrinsic properties of $\mathrm{H} 1$ or its interactors, but also by the modification status of the nucleosomes it associates with. Indeed, Misteli and colleagues showed that the residence time of $\mathrm{H} 1$ molecules on chromatin was greatly decreased when cells were treated with histone deacetylase inhibitors (Misteli et al., 2000). However, the non-specific effects of these general drugs precludes distinguishing whether the increased $\mathrm{H} 1$ dynamics was due to core histone acetylation, acetylation of the linker histone itself or indeed of any other (remodeling) factor. Most likely, all of these factors contribute to $\mathrm{H} 1$ dynamics and assembly. This is illustrated by a recent study by Vaquero and colleagues, who demonstrated that the human histone deacetylase 
SirT1 promoted the $\mathrm{H} 1$ incorporation into facultative heterochromatin by coordinating stepwise deacetylation of H1, H3K9 and H4K16 (Vaquero et al., 2006). This provides in vivo support for the old notion that the folding of the nucleosomal fiber as a prerequisite for the formation of higher order chromatin structure requires, among other factors, the coordinate deacetylation of nucleosomes and $\mathrm{H} 1$ incorporation. Targeting of specific histone modifications may also affect $\mathrm{H} 1$ occupancy very locally and thus contribute to transcriptional regulation. Zhu and colleagues recently found that coordinated $\mathrm{H} 2 \mathrm{~A}$ deubiquitination and histone acetylation led to the release of $\mathrm{H} 1$ from the promoters of androgen receptorregulated genes (Zhu et al., 2007). Histone acetylation is preceded by demethylation of $\mathrm{H} 3$ (Metzger et al., 2006). Whether the dissociation of $\mathrm{H} 1$ is aided by chaperones or SWI/SNF-type remodeling activities known to contribute to nuclear hormone receptor activation (see below) remains to be determined.

\section{Local and global linker histone depletion by remodeling}

The chromatin of most eukaryotic cells contains approximately one linker histone per nucleosome, but this ratio varies between organisms and cell types. Interestingly, the linker-to-core histone ratio is inversely correlated with the degree of cellular differentiation, approaching 1 in highly differentiated cells and found to be considerably lower in less differentiated cells (Woodcock et al., 2006). For example, Fan and colleagues reported that in mammalian stem cells, only one $\mathrm{H} 1$ per two nucleosomes is bound to chromatin (Fan et al., 2005). In preblastoderm stages of frog and fly embryos, somatic $\mathrm{H} 1$ is replaced entirely by the specialized linker histone B4 or HMG-D, respectively, both binding to the nucleosome with a significantly lower affinity than $\mathrm{H} 1$ (Ner and Travers, 1994; Saeki et al., 2005). In flies, these early developmental stages are characterized by largely decondensed chromatin (Ner and Travers, 1994). Because H1 promotes fiber folding and inhibits nucleosome remodeling to some extent (see above), $\mathrm{H} 1$ levels may serve as a measure of chromatin plasticity.

The important conclusion from these observations is that chromatin fibers fully saturated with linker histones may be the exception rather than the rule. If, however, $\mathrm{H} 1$ levels are limited, the next important question that arises is whether the linker histones are randomly distributed or whether their local clustering (which leaves other areas of the chromosome vacant) contributes to the structural and functional differentiation of the nucleus. The latter scenario appears to be the case. Mapping linker histones in embryonic stems cells, Fan and colleagues found linker histone deprivation at a class of imprinted genes (Fan et al., 2005). The principles by which selective incorporation of linker histone is achieved are still mysterious, but as the case of the mouse mammary tumor virus (MMTV) promoter illustrates placement of $\mathrm{H} 1$ at particular nucleosomes may have both positive and negative effects on gene regulation.
It was noted early on that the transcriptional competence of the MMTV promoter (i.e., its glucocorticoid inducibility) is associated with phosphorylation of $\mathrm{H} 1$ at nucleosome B (Lee and Archer, 1998; Koop et al., 2003). This nucleosome bears all binding sites for the transcription activators involved, most prominently for the glucocorticoid or progesterone receptors (GR, PR). The canonical structure of nucleosome $B$ is perturbed by the vertebrate BRG1-containing remodeling complex to allow interaction of all regulators (Vicent et al., 2006). Surprisingly, however, the initial presence of histone $\mathrm{H} 1$ is required for full inducibility of the promoter. Koop and his team have argued that $\mathrm{H} 1$ interaction contributes to the precise positioning of nucleosome $\mathrm{B}$ that assures interaction of PR with its binding site on the nucleosomal surface (Koop et al., 2003). Belikov and colleagues have now confirmed these early findings in a heterologous Xenopus reconstitution system and also found that substoichiometric levels of $\mathrm{H} 1$ are required for optimal gene induction (Belikov et al., 2007). In this work, the activation of the MMTV promoter was studied by reconstituting it in Xenopus oocytes and expressing carefully controlled amounts of GR and H1. As Xenopus oocytes do not contain any somatic histone $\mathrm{H} 1$, but only the variant $\mathrm{B} 4$, the effect of increasing levels of exogenous $\mathrm{H} 1$ on transcriptional regulation could be monitored. By this means, Belikov et al. found that only at substoichiometric concentrations of $\mathrm{H} 1$ a full and efficient activation of the promoter was obtained. Saturating $\mathrm{H} 1$ levels apparently rendered chromatin too rigid to be remodeled. The fact that a particular $\mathrm{H} 1$ density of $\mathrm{H} 1$ is favorable for activation can be explained in local and global terms: globally, a certain amount of $\mathrm{H} 1$ may be required to hide cryptic sites by tight chromatin packaging, which will effectively increase the concentration of GR available for nucleosome $\mathrm{B}$ interaction. Locally, $\mathrm{H} 1$ must initially contribute to positioning of nucleosome B. In contrast, nucleosome $\mathrm{B}$ remodeling, a prerequisite for transcriptional activation, requires the prior release of histone $\mathrm{H} 1$ (Koop et al., 2003; Belikov et al., 2007). Whether H1 release and nucleosome $B$ remodeling are results of separate remodeling events or indicative of one chromatosome remodeling reaction is not known. It is possible that the removal of $\mathrm{H} 1$ marks and unlocks nucleosome $\mathrm{B}$ for full remodeling and thus initiates a series of coordinate reactions that lead to promoter activation (Figure 1c-f).

The case of the MMTV promoter demonstrates the importance of local $\mathrm{H} 1$ binding and dissociation for gene regulation. An interesting case that points to a previously unappreciated relationship between rather global $\mathrm{H} 1$ chromosome interactions, ATP-dependent remodeling and histone modification has recently been provided by Corona and collaborators, who studied mutant Drosophila in which the remodeling ATPase ISWI had been depleted or perturbed by expression of a dominant negative form thereof (Corona et al., 2007). Depletion of ISWI has no obvious global defect on chromosome structure except on the polytenic $\mathrm{X}$ chromosome in male larval salivary glands, which becomes largely deformed and decondensed. In search for an explanation it was found that the $\mathrm{X}$ chromosome appeared to be largely depleted of $\mathrm{H} 1$, while the autosomes showed no obvious $\mathrm{H} 1$ 
reduction. In Drosophila, the male X chromosome is the target of the dosage compensation complex whose activity stimulates transcription of $\mathrm{X}$-linked genes by approximately two-fold (Corona et al., 2007). This involves chromosome-wide acetylation of H4K16, a modification that distinguishes the male $\mathrm{X}$ chromosome from all other chromosomes and obviously sensitizes the chromatin of the $\mathrm{X}$ chromosome for the dissociation of $\mathrm{H} 1$. The phenomenology suggests that an ISWI-containing remodeling factor contributes to the association of $\mathrm{H} 1$ to chromatin. As detailed earlier, the ISWI containing CHRAC/ACF complex is able to promote the assembly of $\mathrm{H} 1$-containing chromatin in vitro. The genetic analysis of the $\mathrm{X}$ chromosome phenotype, however, shows that not CHRAC/ACF but the nucleosome-remodeling factor NURF could be involved (Badenhorst et al., 2002). NURF is an ISWI-containing remodeling factor whose nucleosome sliding activity has so far not been related to chromatin assembly/disassembly, but rather to transcription regulation. It therefore remains unclear whether NURF is directly involved in $\mathrm{H} 1$ deposition, or indirectly, e.g., through effects on gene expression. The selective depletion of $\mathrm{H} 1$ from the $\mathrm{H} 4 \mathrm{~K} 16$ acetylated chromosome suggests that acetylation at this strategic site may weaken the interaction of $\mathrm{H} 1$ with nucleosomes and that a remodeling action may counteract this effect to a certain extent. Conceivably, the H4K16ac mark may lead to a slight depletion of $\mathrm{H} 1$ on the male $\mathrm{X}$ chromosome even in the presence of ISWI. This would be predicted to affect the extent of folding of X-chromosomal chromatin and explain its hyperactive state. Alternatively, the heightened transcriptional status of the $X$ chromosome may lead to enhanced eviction of histones, which may not be properly replaced in the absence of ISWI. In any case, the observation by Corona et al. emphasizes an important functional connection between at least three different players regulating chromosome structure: histone modification, $\mathrm{H} 1$ dynamics and ATP-dependent chromatin remodeling factors.

\section{Concluding remarks}

The consequences of global and local depletion of linker histones emphasize the important role for these molecules for chromatin structure and function. Linker histones restrict spontaneous nucleosome movements and promote chromatin folding, effectively limiting chromatin dynamics and activity. On the other hand, the interactions of $\mathrm{H} 1$ itself with chromatin appear surprisingly dynamic. How can these apparently contradictory properties of $\mathrm{H} 1$ be reconciled? The key to answering this question is the notion that $\mathrm{H} 1$-chromatin interactions are regulated both on a global and on a local level. We have summarized some parameters that govern these interactions. The recent appreciation of the effects of ATP-dependent nucleosome remodeling factors on $\mathrm{H} 1$ turnover or even the movement of entire chromatosomes may explain some of the overt phenomenology. ATPdependent $\mathrm{H} 1$ dynamics appear to be tightly controlled and integrated with all other mechanisms of chromatin regulation. The same principles that control core histones dynamics (intrinsic affinity, variant properties, modification status, specific chaperone interactions and ATPdependent remodeling) can be applied to $\mathrm{H} 1$ as well and thus extend the regulatory complexity. As not all remodeling factors appear to be equally suited to deal with linker histones, $\mathrm{H} 1$ will be a substrate for some factors, but affect the activity of others. We propose that the former factors be called 'chromatin remodeling factors' and be distinguished from the 'nucleosome remodeling factors' whose actions are hindered by the presence of $\mathrm{H} 1$. This distinction is particularly relevant for those prevalent situations in cells, where linker histones are present at substoichiometric levels with respect to nucleosomes. Establishing the chromosomal 'landscape' of $\mathrm{H} 1$ interactions will reveal the full regulatory contribution of linker histones.

\section{Acknowledgments}

Work on ATP-dependent nucleosome remodeling in the Becker laboratory is supported by Deutsche Forschungsgemeinschaft through SFB 594 and by the European Union via the Network of Excellence 'The Epigenome' (FP6-503433). V.K.M. is a fellow of the Munich International Max Planck Research School for Molecular and Cellular Life Sciences.

\section{References}

Anderson, J.D., Thastrom, A., and Widom, J. (2002). Spontaneous access of proteins to buried nucleosomal DNA target sites occurs via a mechanism that is distinct from nucleosome translocation. Mol. Cell. Biol. 22, 7147-7157.

Angelov, D., Bondarenko, V.A., Almagro, S., Menoni, H., Mongelard, F., Hans, F., Mietton, F., Studitsky, V.M., Hamiche, A., Dimitrov, S., et al. (2006). Nucleolin is a histone chaperone with FACT-like activity and assists remodeling of nucleosomes. EMBO J. 25, 1669-1679.

Badenhorst, P., Voas, M., Rebay, I., and Wu, C. (2002). Biological functions of the ISWI chromatin remodeling complex NURF. Genes Dev. 16, 3186-3198.

Becker, P.B. (2002). Nucleosome sliding: facts and fiction. EMBO J. 21, 4749-4753.

Becker, P.B. and Horz, W. (2002). ATP-dependent nucleosome remodeling. Annu. Rev. Biochem. 71, 247-273.

Bednar, J., Horowitz, R.A., Grigoryev, S.A., Carruthers, L.M., Hansen, J.C., Koster, A.J., and Woodcock, C.L. (1998). Nucleosomes, linker DNA, and linker histone form a unique structural motif that directs the higher-order folding and compaction of chromatin. Proc. Natl. Acad. Sci. USA 95, 14173-14178.

Belikov, S., Astrand, C., and Wrange, O. (2007). Mechanism of histone $\mathrm{H} 1$-stimulated glucocorticoid receptor DNA binding in vivo. Mol. Cell. Biol. 27, 2398-2410.

Brown, D.T., Izard, T., and Misteli, T. (2006). Mapping the interaction surface of linker histone $\mathrm{H} 1(0)$ with the nucleosome of native chromatin in vivo. Nat. Struct. Mol. Biol. 13, 250-255.

Catez, F., Ueda, T., and Bustin, M. (2006). Determinants of histone $\mathrm{H} 1$ mobility and chromatin binding in living cells. Nat. Struct. Mol. Biol. 13, 305-310.

Clapier, C.R., Nightingale, K.P., and Becker, P.B. (2002). A critical 
epitope for substrate recognition by the nucleosome remodeling ATPase ISWI. Nucleic Acids Res. 30, 649-655.

Corona, D.F., Siriaco, G., Armstrong, J.A., Snarskaya, N., McClymont, S.A., Scott, M.P., and Tamkun, J.W. (2007). ISWI regulates higher-order chromatin structure and histone $\mathrm{H} 1$ assembly in vivo. PLoS Biol. 5, e232.

Daujat, S., Zeissler, U., Waldmann, T., Happel, N., and Schneider, R. (2005). HP1 binds specifically to Lys26-methylated histone H1.4, whereas simultaneous Ser27 phosphorylation blocks HP1 binding. J. Biol. Chem. 280, 38090-38095.

de la Serna, I.L., Ohkawa, Y., and Imbalzano, A.N. (2006). Chromatin remodeling in mammalian differentiation: lessons from ATP-dependent remodelers. Nat. Rev. Genet. 7, 461-473.

Dimitrov, S. and Wolffe, A.P. (1996). Remodeling somatic nuclei in Xenopus laevis egg extracts: molecular mechanisms for the selective release of histones $\mathrm{H} 1$ and $\mathrm{H} 1(0)$ from chromatin and the acquisition of transcriptional competence. EMBO J. 15, 5897-5906.

Fan, L. and Roberts, V.A. (2006). Complex of linker histone H5 with the nucleosome and its implications for chromatin packing. Proc. Natl. Acad. Sci. USA 103, 8384-8389.

Fan, Y., Nikitina, T., Zhao, J., Fleury, T.J., Bhattacharyya, R., Bouhassira, E.E., Stein, A., Woodcock, C.L., and Skoultchi, A.I. (2005). Histone $\mathrm{H} 1$ depletion in mammals alters global chromatin structure but causes specific changes in gene regulation. Cell 123, 1199-1212.

Flaus, A. and Owen-Hughes, T. (2004). Mechanisms for ATPdependent chromatin remodeling: farewell to the tuna-can octamer? Curr. Opin. Genet. Dev. 14, 165-173.

Hill, D.A. and Imbalzano, A.N. (2000). Human SWI/SNF nucleosome remodeling activity is partially inhibited by linker histone $\mathrm{H} 1$. Biochemistry 39, 11649-11656.

Horn, P.J., Carruthers, L.M., Logie, C., Hill, D.A., Solomon, M.J., Wade, P.A., Imbalzano, A.N., Hansen, J.C., and Peterson, C.L. (2002). Phosphorylation of linker histones regulates ATPdependent chromatin remodeling enzymes. Nat. Struct. Biol. 9, 263-267.

Ito, T., Levenstein, M.E., Fyodorov, D.V., Kutach, A.K., Kobayashi, R., and Kadonaga, J.T. (1999). ACF consists of two subunits, Acf1 and ISWI, that function cooperatively in the ATPdependent catalysis of chromatin assembly. Genes Dev. 13, 1529-1539.

Izzo, A., Kamieniarz, K., and Schneider, R. (2008). The histone $\mathrm{H} 1$ family: specific members, specific functions? Biol. Chem. 389, 333-343.

Karetsou, Z., Sandaltzopoulos, R., Frangou-Lazaridis, M., Lai, C.Y., Tsolas, O., Becker, P.B., and Papamarcaki, T. (1998). Prothymosin $\alpha$ modulates the interaction of histone $\mathrm{H} 1$ with chromatin. Nucleic Acids Res. 26, 3111-3118.

Koop, R., Di Croce, L., and Beato, M. (2003). Histone H1 enhances synergistic activation of the MMTV promoter in chromatin. EMBO J. 22, 588-599.

Langst, G. and Becker, P.B. (2001). Nucleosome mobilization and positioning by ISWI-containing chromatin-remodeling factors. J. Cell Sci. 114, 2561-2568.

Lee, H.L. and Archer, T.K. (1998). Prolonged glucocorticoid exposure dephosphorylates histone $\mathrm{H} 1$ and inactivates the MMTV promoter. EMBO J. 17, 1454-1466.

Lorch, Y., Maier-Davis, B., and Kornberg, R.D. (2006). Chromatin remodeling by nucleosome disassembly in vitro. Proc. Natl. Acad. Sci. USA 103, 3090-3093.

Loyola, A. and Almouzni, G. (2004). Histone chaperones, a supporting role in the limelight. Biochim. Biophys. Acta 1677, 3-11.

Lusser, A., Urwin, D.L., and Kadonaga, J.T. (2005). Distinct activities of CHD1 and ACF in ATP-dependent chromatin assembly. Nat. Struct. Mol. Biol. 12, 160-166.

Maier, V.K., Chioda, M., Rhodes, D., and Becker, P.B. (2007). ACF catalyses chromatosome movements in chromatin fibres. EMBO J., Epub ahead of print; DOI: 10.1038/ sj.emboj.7601902.
Mazurkiewicz, J., Kepert, J.F., and Rippe, K. (2006). On the mechanism of nucleosome assembly by histone chaperone NAP1. J. Biol. Chem. 281, 16462-16472.

Mellor, J. (2005). The dynamics of chromatin remodeling at promoters. Mol. Cell 19, 147-157.

Meshorer, E., Yellajoshula, D., George, E., Scambler, P.J., Brown, D.T., and Misteli, T. (2006). Hyperdynamic plasticity of chromatin proteins in pluripotent embryonic stem cells. Dev. Cell 10, 105-116.

Metzger, E., Wissmann, M., and Schule, R. (2006). Histone demethylation and androgen-dependent transcription. Curr. Opin. Genet. Dev. 16, 513-517.

Misteli, T., Gunjan, A., Hock, R., Bustin, M., and Brown, D.T. (2000). Dynamic binding of histone $\mathrm{H} 1$ to chromatin in living cells. Nature 408, 877-881.

Ner, S.S. and Travers, A.A. (1994). HMG-D, the Drosophila melanogaster homologue of HMG 1 protein, is associated with early embryonic chromatin in the absence of histone $\mathrm{H} 1$. EMBO J. 13, 1817-1822.

Orrego, M., Ponte, I., Roque, A., Buschati, N., Mora, X., and Suau, P. (2007). Differential affinity of mammalian histone $\mathrm{H} 1$ somatic subtypes for DNA and chromatin. BMC Biol. 5, 22.

Pennings, S., Meersseman, G., and Bradbury, E.M. (1994). Linker histones $\mathrm{H} 1$ and $\mathrm{H} 5$ prevent the mobility of positioned nucleosomes. Proc. Natl. Acad. Sci. USA 91, 10275-10279.

Ramachandran, A., Omar, M., Cheslock, P., and Schnitzler, G.R. (2003). Linker histone $\mathrm{H} 1$ modulates nucleosome remodeling by human SWI/SNF. J. Biol. Chem. 278, 48590-48601.

Richardson, R.T., Alekseev, O.M., Grossman, G., Widgren, E.E., Thresher, R., Wagner, E.J., Sullivan, K.D., Marzluff, W.F., and O'Rand, M.G. (2006). Nuclear autoantigenic sperm protein (NASP), a linker histone chaperone that is required for cell proliferation. J. Biol. Chem. 281, 21526-21534.

Robinson, P.J. and Rhodes, D. (2006). Structure of the ' $30 \mathrm{~nm}$ ' chromatin fibre: a key role for the linker histone. Curr. Opin. Struct. Biol. 16, 336-343.

Saeki, H., Ohsumi, K., Aihara, H., Ito, T., Hirose, S., Ura, K., and Kaneda, Y. (2005). Linker histone variants control chromatin dynamics during early embryogenesis. Proc. Natl. Acad. Sci. USA 102, 5697-5702.

Saha, A., Wittmeyer, J., and Cairns, B.R. (2006). Chromatin remodeling: the industrial revolution of DNA around histones. Nat. Rev. 7, 437-447.

Shogren-Knaak, M. and Peterson, C.L. (2006). Switching on chromatin: mechanistic role of histone H4-K16 acetylation. Cell Cycle 5, 1361-1365.

Tyler, J.K. (2002). Chromatin assembly. Cooperation between histone chaperones and ATP-dependent nucleosome remodeling machines. Eur. J. Biochem. 269, 2268-2274.

Vaquero, A., Scher, M.B., Lee, D.H., Sutton, A., Cheng, H.L., Alt, F.W., Serrano, L., Sternglanz, R., and Reinberg, D. (2006). $\mathrm{SirT2}$ is a histone deacetylase with preference for histone $\mathrm{H} 4$ Lys 16 during mitosis. Genes Dev. 20, 1256-1261.

Varga-Weisz, P.D., Blank, T.A., and Becker, P.B. (1995). Energydependent chromatin accessibility and nucleosome mobility in a cell-free system. EMBO J. 14, 2209-2216.

Vicent, G.P., Ballare, C., Zaurin, R., Saragueta, P., and Beato, M. (2006). Chromatin remodeling and control of cell proliferation by progestins via cross talk of progesterone receptor with the estrogen receptors and kinase signaling pathways. Ann. N.Y. Acad. Sci. 1089, 59-72.

Wisniewski, J.R., Zougman, A., Kruger, S., and Mann, M. (2007). Mass spectrometric mapping of linker histone $\mathrm{H} 1$ variants reveals multiple acetylations, methylations, and phosphorylation as well as differences between cell culture and tissue. Mol. Cell. Proteomics 6, 72-87.

Woodcock, C.L., Skoultchi, A.I., and Fan, Y. (2006). Role of linker histone in chromatin structure and function: $\mathrm{H} 1$ stoichiometry and nucleosome repeat length. Chromosome Res 14, 17-25. 
Workman, J.L. (2006). Nucleosome displacement in transcription. Genes Dev. 20, 2009-2017.

Yellajoshyula, D. and Brown, D.T. (2006). Global modulation of chromatin dynamics mediated by dephosphorylation of linker histone $\mathrm{H} 1$ is necessary for erythroid differentiation. Proc. Natl. Acad. Sci. USA 103, 18568-18573.

Zhu, P., Zhou, W., Wang, J., Puc, J., Ohgi, K.A., Erdjument-
Bromage, H., Tempst, P., Glass, C.K., and Rosenfeld, M.G. (2007). A histone H2A deubiquitinase complex coordinating histone acetylation and $\mathrm{H} 1$ dissociation in transcriptional regulation. Mol. Cell 27, 609-621.

Zlatanova, J., Caiafa, P., and Van Holde, K. (2000). Linker histone binding and displacement: versatile mechanism for transcriptional regulation. FASEB J. 14, 1697-1704. 